\title{
Inhibition of Penicillin Transport from the Cerebrospinal Fluid after Intracisternal Inoculation of Bacteria
}

\author{
ReYnold Spector and A. V. Lorenzo \\ From the Department of Neurology, The Children's Hospital Medical Center, \\ Department of Medicine, Peter Bent Brigham Hospital; and the Departments of \\ Medicine and Pharmacology, Harvard Medical School, \\ Boston, Massachusetts 02115
}

A в S T R A C T The effect of intracisternal inoculation of bacteria on the choroid plexus system, which transports penicillin from cerebrospinal fluid (CSF) to blood, was studied in vitro and in vivo. Meningeal and choroid plexus inflammations as well as CSF pleocytosis were induced in rabbits with intracisternal inoculations of Hemophilus influenzae or Staphylococcus aureus. At various times after bacterial inoculation, the choroid plexuses of the inoculated rabbits were removed and incubated in artificial CSF containing $\left[{ }^{14} \mathrm{C}\right]$ penicillin. The ability of the choroid plexuses to accumulate pencillin in vitro was measured and was found to be depressed as compared with controls. This depression of choroid plexus uptake reversed with resolution of the inflammatory process. In vivo on the day after intracisternal inoculation of Hemophilus influenzae, a decrease in the disappearance of penicillin relative to inulin in the inoculated rabbits (as compared to the controls) was observed when $\left[{ }^{14} \mathrm{C}\right]$ penicillin and $\left[{ }^{3} \mathrm{H}\right]$ inulin were injected intraventricularly and cisternal CSF was sampled $2 \mathrm{~h}$ later. This decrease could not be explained by penicillin binding to the CSF exudate. However, the choroid plexus transport system for penicillin was only partially depressed in those inoculated rabbits with bacterially induced inflammation, since in vitro the choroid plexuses could still accumulate penicillin and in vivo CSF penicillin levels could be further increased with probenecid pretreatment. These results suggest that CSF penicillin levels are increased in this model due to three factors: a depression of active efflux of penicillin from the CSF, an increase in permeability to penicillin of inflamed meninges, and, less significantly, by CSF binding of penicillin.

Dr. Lorenzo is a recipient of National Institutes of Health Career Development Award HD18519.

Received for publication 2 October 1973 and in revised form 7 January 1974.

\section{INTRODUCTION}

In both human and experimental meningitis, an increased permeability of molecules of various types from blood to cerebrospinal fluid $(\mathrm{CSF})^{1}$ has been documented $(1,2)$. This permeability change has been attributed to inflammatory changes in the membranes that comprise the "blood-CSF" barrier $(1,2)$. This nonspecific increase in the permeability of the bloodCSF barrier in meningitis has been assumed to be the cause of the increased CSF concentrations of certain drugs (like penicillin) (2-4). However, Harter and Petersdorf (2) suggested that another factor, viz., an inhibition of efflux from CSF to blood in meningitis, might affect drug distribution. This perceptive insight was based on an analysis of phenolsulphonphthalein (PSP) transport from the CSF in meningitis in humans (5). Subsequently, the active transport of PSP (6) and other substances such as penicillin (7-9) from the CSF was documented. If the hypothesis of Harter and Petersdorf (2) is extended to include the hypothesis that there is depression of active transport from the CSF compartment in meningitis, a portion of the elevated levels of drugs actively transported from the CSF could be due to a depression of the efflux transport systems. There is evidence that carrier-mediated transport (facilitated diffusion) of glucose from blood to CSF is depressed in experimental meningitis (1).

The purpose of this study was to show (a) that, after the intracisternal inoculation of bacteria, a portion of the increase in CSF levels of penicillin is due to depression of the efflux of penicillin from the CSF compartment $(b)$ that this is due, in part, to depression of

${ }^{1}$ Abbreviations used in this paper: CSF, cerebrospinal fluid; H. flu., Hemophilus influenzae; HSA, human serum albumin; PBS, phosphate-buffered saline; PSP, phenolsulfonphthalein; $\mathrm{T} / \mathrm{M}$, tissue-to-medium ratio. 
the choroid plexus carrier mechanism (c) that, although depressed, the choroid plexus is still able to transport penicillin from CSF and $(d)$ that this residual transport capability can be further depressed by probenecid with a resultant increase in CSF/plasma penicillin ratios.

To investigate these points, we employed two rabbit models that involved direct inoculation of bacteria into the CSF. Although pathogenetically unrelated to human meningitis, these models yielded levels of CSF protein, cellular exudate, and penicillin similar to those seen in human meningitis. In one model, inoculation of Hemophilus influenzae yielded a mild clinical illness that resolved without antibiotic therapy. On the other end of the spectrum, in the second model, inoculation of a virulent strain of Staphylococcus aureus resulted in a rapidly fatal meningitis with septicemia. These two models allowed us to investigate penicillin passage into and out of the CSF at both extremes of the clinical spectrum.

\section{METHODS}

The following radiochemicals were employed: [25I]human serum albumin (HSA; $1 \mu \mathrm{Ci} / \mathrm{mg}$ ) from Mallinckrodt Chemical Works, St. Louis, Mo.; $\left[{ }^{14} \mathrm{C}\right]$ benzyl penicillin (38$42 \mathrm{mCi} / \mathrm{mM}$ ) from Amersham/Searle Corp., Arlington Heights, Ill.; $\left[{ }^{3} \mathrm{H}\right]$ inulin $(0.25 \mathrm{mCi} / 2.4 \mathrm{mg})$ and $\left[{ }^{3} \mathrm{H}\right]-$ mannitol $(3 \mathrm{Ci} / \mathrm{mM}$ ) from New England Nuclear, Boston, Mass.

The models. 21 New Zealand white rabbits, weighing approximately $2.0 \mathrm{~kg}$, were anesthetized with intravenous thiopental. Each animal, in this series of rabbits as well as in the experiments described below, had approximately 0.2 ml CSF withdrawn atraumatically from the cisterna magna unless otherwise noted. Subsequently, each rabbit was injected intracisternally with $0.2 \mathrm{ml}$ of phosphate-buffered saline with $0.1 \%$ gelatin (PBS) and $5 \times 10^{7}$ Hemophilus influenzae type b (H. flu. $)(n=13)$, or approximately $10^{8}$ Staphylococcus aureus (S. aureus) $(n=5)$, or no organisms $(n=3) \quad(10,11)$. The rabbits awoke within $30 \mathrm{~min}$. Rabbits whose CSF was bloody to the unaided eye were not used. The unaided eye can easily recognize a CSF hematocrit of 0.5 . At $18 \mathrm{~h}$ or 3 days after the intracisternal inoculation, the rabbits were reanesthetized with thiopental, and sacrificed with a heart puncture, and 0.8 to $1.2 \mathrm{ml}$ of CSF was withdrawn (9). This technique allowed consistent withdrawal of blood-free CSF, for a large portion of the blood volume entered the chest. The few rabbits whose CSF was bloody to the unaided eye were not used in this study. The blood and CSF were cultured and gram-stained and white blood cell counts and protein determinations (12) were performed on the CSF. Choroid plexuses from four rabbits inoculated with $H$. $f l u$., two rabbits inoculated with $S$. $a u$ reus, two rabbits inoculated with PBS alone, and two uninoculated rabbits from whom CSF was not withdrawn were removed the day after inoculation, placed in $10 \%$ buffered formaldehyde, and subsequently stained with hematoxylin and eosin.

Distribution studies on unanesthetized rabbits. In this series of studies to measure how mannitol and penicillin distribute themselves between CSF and plasma, 24 rabbits were employed. 11 were inoculated with PBS and 13 with
$H$. $f u$. as described under the section above. To show that the $H$. $f u$. model yielded mannitol distributions in CSF and plasma similar to those reported by others, percutaneous intravenous lines were placed into both marginal ear veins of unanesthetized rabbits which had been inocu'ated intracisternally the previous day ( $18 \mathrm{~h}$ before) with $0.2 \mathrm{ml}$ PBS $(n=6)$ or $5 \times 10^{7} \mathrm{H}$. $f u$. in $0.2 \mathrm{ml} \mathrm{PBS}(n=5) .30 \mu \mathrm{Ci}$ $\left[{ }^{3} \mathrm{H}\right]$ mannitol was dissolved in $15 \mathrm{ml}$ artificial CSF (9), and $4 \mathrm{ml}$ of this solution was infused i.v. over $10 \mathrm{~min}$, and the remainder over $3 \mathrm{~h}$ at a constant rate. At $1.5 \mathrm{~h}$, plasma was obtained from the noninfused ear vein of each rabbit. At $3 \mathrm{~h}, 40 \mathrm{mg} / \mathrm{kg}$ pentobarbital was infused rapidly through the noninfusion i.v. line to cause a respiratory arrest, $20 \mathrm{ml}$ of blood was obtained by cardiac puncture. the heart was severed through the chest wall and $0.8-1.2 \mathrm{ml} \mathrm{CSF}$ was immediately obtained. After CSF sampling, the brain was removed as rapidly as possible and the choroid plexuses from the fourth and the two lateral ventricles were removed, pooled, weighed, and homogenized in $0.5 \mathrm{ml} \mathrm{H}_{2} \mathrm{O}$. The ${ }^{3} \mathrm{H}$ activity in $0.2 \mathrm{ml} \mathrm{CSF}$, choroid plexus homogenate, and plasma was measured by scintillation counting (9).

A similar series of experiments was performed on four $H$. $f u$.-inoculated and five control (PBS-injected) rahbits the day after inoculation, except that $100 \mathrm{mg} / \mathrm{kg}$ sodium penicillin G (E. R. Squibh \& Sons, Princeton, N. J.) was dissolved in $20 \mathrm{ml}$ artificial $\mathrm{CSF}$, and $3 \mathrm{ml}$ was injected initially i.v., and the rest over the remainder of the $3 \mathrm{~h}$. Four other $H$. $f u$.-inoculated rahhits were pretreated $30 \mathrm{~min}$ before the i.v. infusion with $200 \mathrm{mg} / \mathrm{kg}$ i.p. probenecid (Merck Sharp \& Dohme, Merck Co., Inc., West Point. $\mathrm{Pa}$.). The nonpobenecid-treated $H$. $f(u$.-inoculated animals and controls received saline i.p. (9). The penicillin concentration in the CSF, plasma, and ultrafiltrate of plasma was measured by the Sarcina lutea disk method $(9,13)$. A separate standard curve was necessary for the plasma determinations because plasma and penicillin yielted $17 \%$ higher values than penicillin standards in artificial CSF alone.

In vitro studies. In this series of experiments, designed to measure the penicillin uptake by the isolated choroid plexus as well as the CSF binding of penicillin, 31 new rahbits were utilized. 6 rabbits were used as uninjected controls, 5 were inoculated with PBS. 5 with $S$. aureus, and 15 with $H$. $f u$. The CSF from $8 H$. flu.-inoculated rahbits in the distribution studies was also utilized. To see if the isolated choroid plexuses from these rahbits retained the ability to concentrate $\left[{ }^{14} \mathrm{Clpenicillin}\right.$. tissue-to-medium ratios $(\mathrm{T} / \mathrm{M})$ were obtained for choroid plexuses removed from the fourth (one) and from the lateral ventricles (two) of each rabbit $(9,14)$. These rabbits had been divided into six groups. Groups I $(n=6)$ and II $(n=5)$ were uninoculated and PBS-injected controls, respectively. Group III $(n=5)$ were $S$. aureus-inoculated. The rabbits in groups I and II were sacrificed at $18 \mathrm{~h}$ and those in group III, $12 \mathrm{~h}$ after inoculation. Groups IV $(n=6), \mathrm{V}(n=3)$, and VI $(n=2)$ were $H$. $f u$. inoculated and sacrificed at $18 \mathrm{~h}, 3$, and 5 days, respectively, after the intracisternal inoculation. The rabbits were killed at these times after inoculation with an i.v. bolus of pentobarbital, the heart was punctured, and the brain was quickly removed. Three choroid plexuses were removed from each animal and each individual choroid plexus was placed in a flask containing 3 $\mathrm{ml}$ incubating medium (9), $\left[{ }^{14} \mathrm{C}\right]$ penicillin $(0.001 \mathrm{mM})$ and $\left[{ }^{3} \mathrm{H}\right]$ inulin. The incubating medium was placed in a Dubnoff metabolic shaker at $37^{\circ} \mathrm{C}$ in an atmosphere of $95 \% \quad \mathrm{O}_{2}$ and $5 \% \mathrm{CO}_{2}$ for at least $10 \mathrm{~min}$ before the choroid plexus was 
added, and remained there for the period of the incubation. At the end of the incubation, the weight and radioactivity content of the choroid plexuses were assayed $(9,14) . T / M$ were obtained by dividing the disintegrations per minute per milliliter of intracellular water by the disintegrations per minute per milliliter of incubating medium $(9,14)$. At the end of the incubation in two experiments with $H$. $f$ lu.inoculated choroid plexuses, the medium was chromatographed on silica gel $G$ thin layer plates in two solvent systems: $1: 19:$ : acetic acid:acetone and $7: 3::$ isopropanol: methanol as previously described $(9,15,16) .92 \%(n=2)$ and $94 \%(n=2)$ of the ${ }^{14} \mathrm{C}$ activity was associated with the penicillin spot. When $\left[{ }^{14} \mathrm{C}\right]$ penicillin from the manufacturer was dissolved in artificial CSF, spotted directly on thin layer plates (silica gel), and run in these two solvent systems, 95\% (acetic acid:acetone) and 98\% (isopropanol: methanol) of the ${ }^{14} \mathrm{C}$ was penicillin (9). In a previous study, we had shown that more than $84 \%$ of the ${ }^{14} \mathrm{C}$ activity in choroid plexus was associated with penicillin (9).

A second series of in vitro experiments was performed to measure the binding of penicillin to the CSF exudate of rabbits inoculated the previous day with $H$. $f u$. intracisternally. These binding determinations were performed on three sets of rabbits. The first set of three $H$. $f u$.-inoculated rabhits received $200 \mathrm{mg} / \mathrm{kg}$ probenecid before $33 \mathrm{mg} /$ $\mathrm{kg}$ penicillin by constant infusion over $3 \mathrm{~h}$. These rabbits were referred to above under Distribution studies. At the end of the infusion, the CSF from the inoculated rabbits was removed and the penicillin concentration in the CSF and ultrafiltrate of CSF was determined by the Sarcina lutea technique described below. The second set of experiments was identical to the first, except that the four rabbits received no probenecid pretreatment and received $100 \mathrm{mg} / \mathrm{kg}$ penicillin by constant infusion over $3 \mathrm{~h}$ and were the same rabbits as described above under Distribution studies. The third set of four $H$. flu.-inoculated rabbits received no penicillin before the CSF was removed. To the $0.8-1.0 \mathrm{ml}$ of purulent CSF from each rabbit in each set, $0.4 \mathrm{ml}$ of artificial CSF was added. To the CSF of the third set of rabbits. $2 \mu \mathrm{g}\left[{ }^{14} \mathrm{C}\right.$ penicillin was also added. In all three sets, the CSF was then filtered through an ultrafilter (Millipore Corp., Bedford, Mass.: 25.000 PSED (01310) in a stirred $2.5-\mathrm{ml}$ chamber at $37^{\circ} \mathrm{C}$ with $5 \% \quad \mathrm{CO}_{2}: 95 \% \quad \mathrm{O}_{2}$ at 5 lbs. pressure $(9,17)$. The first $0.2 \mathrm{ml}$ of protein-free ultrafiltrate was discarded and the subsequent $0.2 \mathrm{ml}$ was collected for assay. In all three sets, both the ultrafiltrate and narent solution were assayed in triplicate for penicillin by the Sarcina lutea technique $(9.13)$. In the third set of rabbits, the ${ }^{14} \mathrm{C}$ activity in duplicate $0.05-\mathrm{ml}$ aliquots of parent solution and ultrafiltrate were assayed, and the ultrafiltrate was chromatographed in the acetone: acetic acid solvent system on thin layer chromatographic plates as described above $(9,15,16)$. The ultrafiltrate of CSF in the third set contained $77 \pm 5 \% \quad(n=4){ }^{14} \mathrm{C}$ as penicillin, whereas an ultrafiltrate of artificial CSF with $2 \mu \mathrm{g}\left[{ }^{14} \mathrm{C}\right]-$ penicillin added to it and handled in an identical manner contained $85 \pm 2 \% \quad(n=6){ }^{14} \mathrm{C}$ as penicillin. Corrections were made for the amount of ${ }^{14} \mathrm{C}$ not recovered as penicillin, as determined chromatographically, and for a $12 \%$ average loss of recovery in the ultrafiltrate due to nonspecific binding by the filter (17). Because there was no significant difference obtained in the penicillin binding by CSF by the radiochemical versus the microbiological technique nor between the three sets employing the microbiological technintue, the values were averaged. For example, in the four CSF samples to which $\left[{ }^{14} \mathrm{C}\right]$ penicillin was added, the con- centration of penicillin in the ultrafiltrates was $1.24 \pm 0.15$ (SEM) (microbiological) versus $1.41 \pm 0.09$ (SEM) (radiochemical) $\mu \mathrm{g} / \mathrm{ml}$ in the same samples. The percent of penicillin unbound was $72 \pm 11$ (SEM) and $73 \pm 4$ (SEM), respectively, in these four.

Clearance studies. In this series of studies designed to measure penicillin efflux from the CSF, 20 new rabbits were utilized; 9 were inoculated with PBS and 11 with $H$. $f l u$. as described above under Models. Penicillin clearance from the CSF compartment was determined by two methods. In 10 rabbits, ventriculocisternal perfusions were performed on $6 \mathrm{H}$. flu. and 4 control (PBS-injected) rabbits (the day after intracisternal inoculation) by standard methods $(6,9)$. It was recognized in advance that the perfusion technique would wash out the exudate in these rabbits and that only irreversible changes would be measured. After induction of anesthesia, needles were placed in the left lateral ventricle and cisterna magna $(6,9,18)$. The perfusate consisted of $0.3 \mu \mathrm{Ci} / 100 \mathrm{ml}$ [ $\left.{ }^{125} \overline{1}\right] \mathrm{HSA}$ and $0.001 \mathrm{mM}$ $\left[{ }^{14} \mathrm{C}\right]$ penicillin in artificial CSF (9). The perfusate, after equilibration with $5 \% \quad \mathrm{CO}_{2}$, was infused at a constant rate through the ventricular needle at $0.077 \mathrm{ml} / \mathrm{min}$ until three successive 1-ml cisternal outflow aliquots showed constant $\left[{ }^{125} \mathrm{I}\right] \mathrm{HSA}$ activity, thus indicating steady state. ${ }^{14} \mathrm{C}$ and ${ }^{125} \mathrm{I}$ activity were determined in duplicate aliquots of inflow and outflow perfusate, and penicillin clearance and the rate of CSF formation were also determined (18). It was noted that the second and third $1-\mathrm{ml}$ aliquots of perfusate from the $H$. $f u$--inoculated animals were cloudy, but the CSF became clear subsequently. In two of the six ventriculocisternal perfusion experiments on $H$. $f u$.-inoculated rabbits, the CSF outflow during the steady state was chromatographed in the two systems alluded to above to be certain that the $\left[{ }^{14} \mathrm{C}\right.$ penicillin was not being destroyed. More than $95 \%$ of the ${ }^{14} \mathrm{C}$ was associated with penicillin in both systems on these two determinations.

Since anesthesia has been documented to depress eiffux of ions and bases from the CSF during ventriculocisternal perfusions (9), a modification of the Prockop and Fishman technique was employed (1) to test whether inoculation of $H$. $f u$. into the CSF depressed penicillin efflux. Briefly, 10 rabbits, which had been inoculated intracisternally the previous day with either $H$. $f u$. $(n=5)$ or PBS $(n=5)$, were anesthetized with thiopental. A hole was made in the skill over the left lateral ventricle with a dental drill. A solution of $0.2 \mathrm{ml}$ of artificial CSF with $4 \mu \mathrm{g}\left[{ }^{14} \mathrm{C}\right]$ penicillin and 6 $\mu \mathrm{Ci}\left[{ }^{3} \mathrm{H}\right]$ inulin was injected into the left lateral ventricle stereotactically. Immediately thereafter, the hole in the skull was sealed with bone wax and the skin sutured. The rabbit awoke within approximately $30 \mathrm{~min} .2 \mathrm{~h}$ after the injection, the conscious animal was reanesthetized and killed with a cardiac puncture, and cisternal CSF $(0.4-0.8 \mathrm{ml})$ was sampled. Duplicate aliquots of the injected solution $(0.1$ $\mathrm{ml})$ and the CSF $(0.2-0.4 \mathrm{ml})$ were counted (9). The ratio of the ${ }^{14} \mathrm{C} /{ }^{3} \mathrm{H}$ radioactivity in the $\mathrm{CSF}$ was divided by the ratio of the ${ }^{14} \mathrm{C} /{ }^{3} \mathrm{H}$ radioactivity in the injected solution and multiplied by 100 in both the control and meningitic rabbits. In two $H$. flu.-inoculated and two control rabbits, the CSF was spun down at $5,000 \mathrm{~g}$ for $10 \mathrm{~min}$. The supernate was spotted on silica gel $G$ plates and run in the isopropanol: methanol system noted above. In all four animals, more than $90 \%$ of the ${ }^{14} \mathrm{C}$ was penicillin. Similarly, $5-\mu 1$ duplicates of CSF supernate of two $H$. $f u$.-inoculated and two control rabbits were spotted on Whatman filter paper and run $10 \mathrm{~cm}$ in $n$-butanol: ethanol: $\mathrm{H}_{2} \mathrm{O}:: 52: 18: 30$. The paper was cut in $101-\mathrm{cm}$ pieces and counted. Similarly, 


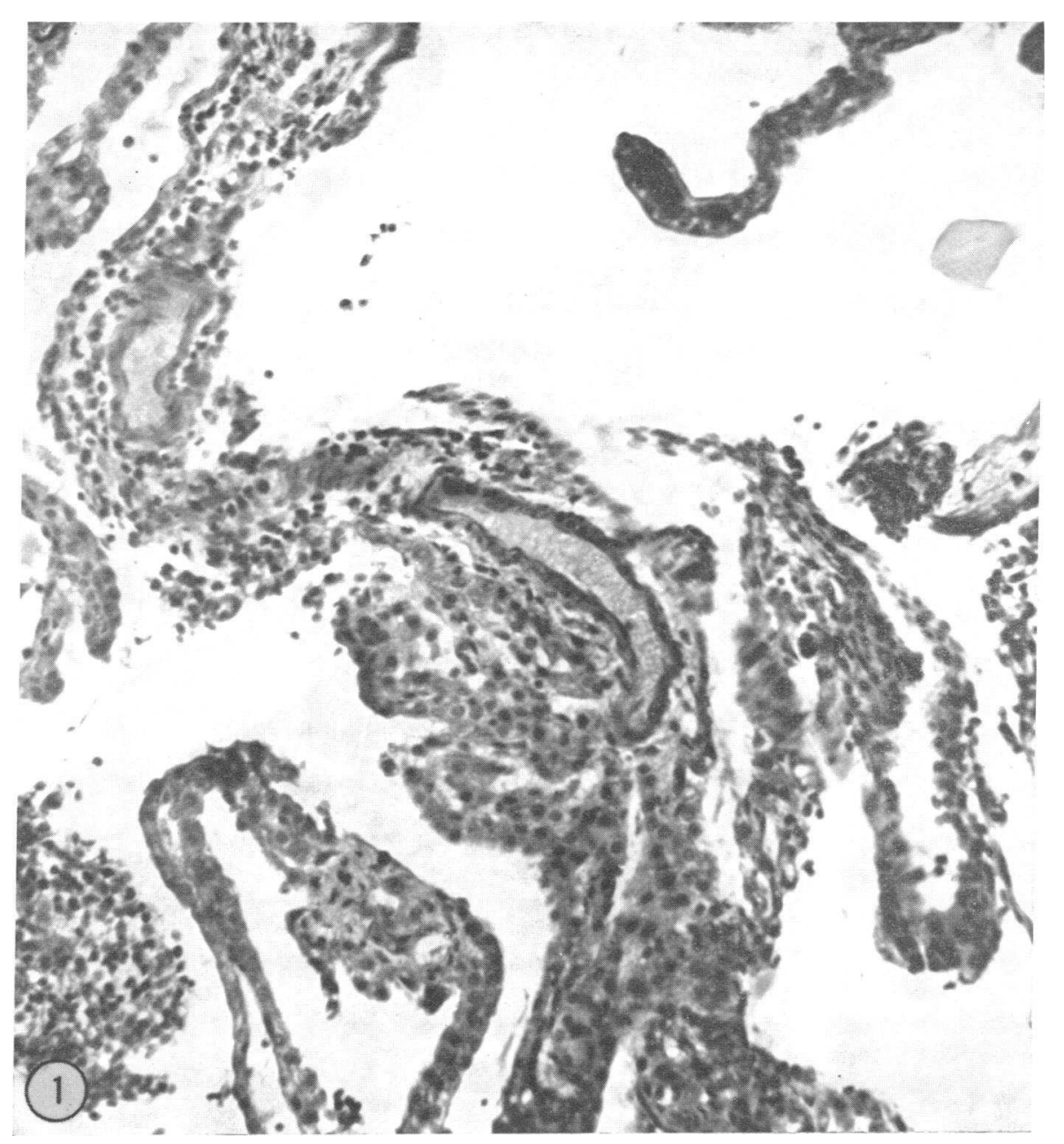

Figure 1 The fourth ventricular choroid plexus was obtained $20 \mathrm{~h}$ after the intracisternal inoculation of $5 \times 10^{7} \mathrm{H}$. influenzae. The choroid plexus was significantly infiltrated with inflammatory cells. Hematoxylin and eosin stain. $\times 230$.

$\left[{ }^{3} \mathrm{H}\right]$ inulin $(0.1 \mu \mathrm{Ci})$ was spotted directly as purchased from the company. In all cases more than $98 \%$ of the ${ }^{3} \mathrm{H}$ activity was at the origin. (The chromatographic system was obtained from New England Nuclear.)

\section{RESULTS}

The models. Rabbits injected with $5 \times 10^{7} \mathrm{H}$. flu. appeared sluggish the day after inoculation but did not have stiff necks. By 3 days, the rabbits appeared normal. The CSF white cell count the day after injection was $25,155 \pm 5,878 \mathrm{~mm}^{3}(\operatorname{SEM} n=9)$ and the CSF protein was $358 \pm 102 \mathrm{mg} / 100 \mathrm{ml}(\mathrm{SEM} ; n=10)$. By the third day after injection, the CSF white cell count had fallen to $8,700 \pm 1,522$ ( $\mathrm{SEM} ; n=3$ ). Although pleomorphic gram-negative rods were visible on gram stain the day after injection, all CSF $(n=10)$ and blood cultures $(n=4)$ were negative. This $H$. $f u$. model we term a model of resolving meningeal inflammation. Control animals injected with PBS appeared normal the day after injection and had a CSF white cell count of 183 109 (SEM; $n=3$ ) and a CSF protein of $53 \pm 3$ (SEM; $n=3$ ). Figs. $1-3$ are photomicrographs of the fourth ventricular choroid plexuses of animals injected with $H$. $f u$., PBS, or $S$. aureus and sacrificed the day after inoculation.

The $S$. aureaus model contrasted sharply with the $H$. $f l u$. model. After a short period of appearing well, five rabbits inoculated with $\mathrm{S}$. aureus rapidly deteriorated and died within $20 \mathrm{~h}$ of the injection. CSF and blood cultures in four of the four rabbits (which were also used to measure choroid plexus uptake of $\left[{ }^{14} \mathrm{C}\right]$ penicillin) were positive for $S$. aureus when obtained $12 \mathrm{~h}$ after intracisternal inoculation. 

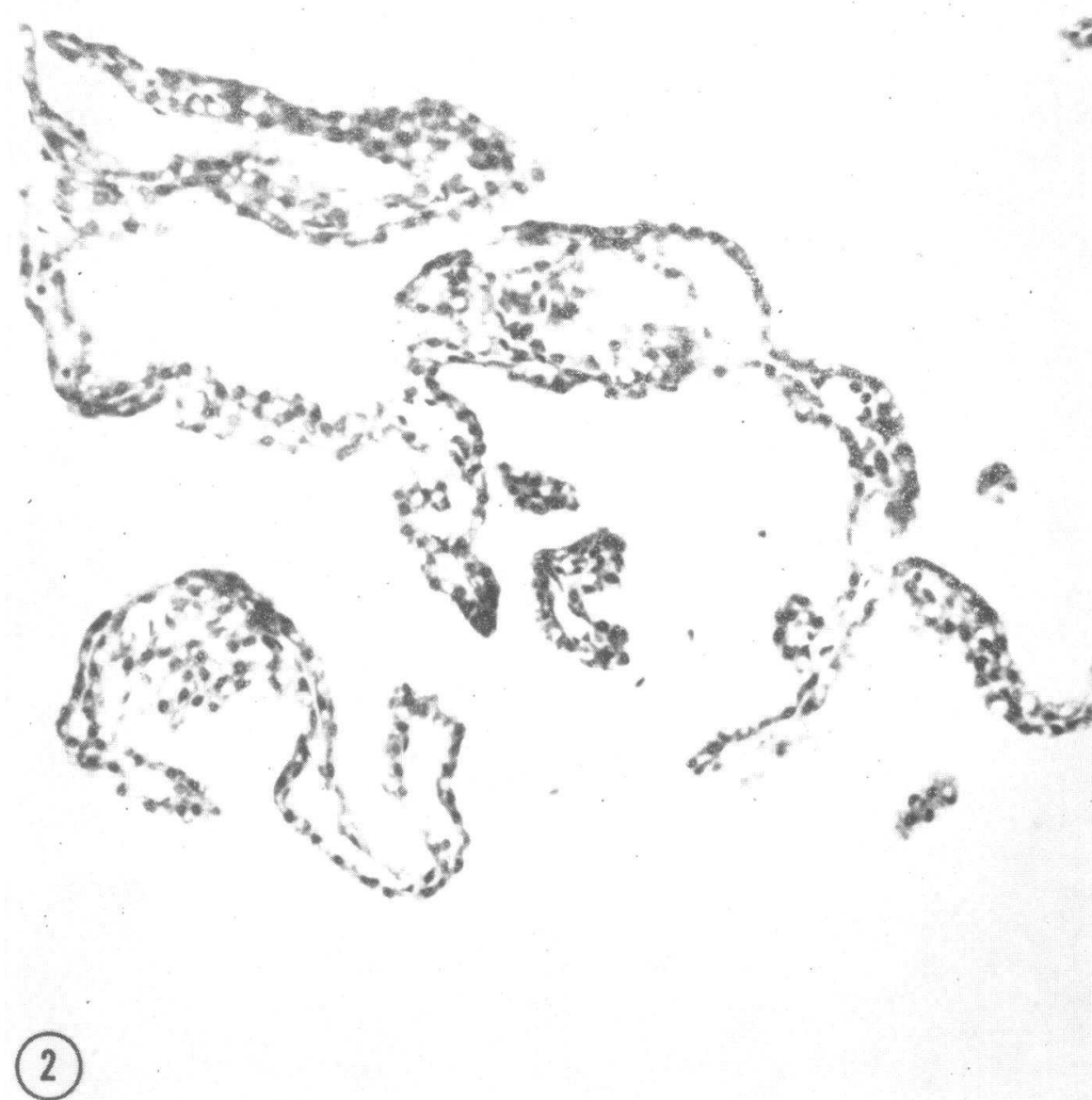

FIgURE 2 The fourth ventricular choroid plexus was obtained $20 \mathrm{~h}$ after the intracisternal injection of PBS. The choroid plexus appeared normal. Hematoxylin and eosin stain. $\times 230$.

In vivo studies. The levels of mannitol relative to plasma in CSF and choroid plexus after a constant 3-h intravenous infusion are shown in Table I. The level of mannitol in CSF of the $H$. $f u$.-inoculated rabbits was significantly higher $(P<0.05)$ than controls.

Penicillin concentrations within CSF and ultrafilterable plasma after a 3 -h constant infusion are shown in Table II. As noted by others, the CSF/plasma penicillin ratio increased in the $H$. $f u$.-inoculated rabbits $(2,11$, 19). Probenecid pretreatment caused a striking increase in CSF penicillin even when only one-third the dose of penicillin was given intravenously (to maintain comparable plasma levels).

In vitro studies. The $5-\mathrm{min} \mathrm{T} / \mathrm{M}$ ratios of the choroid plexuses for penicillin in the various groups of control and inoculated animals are illustrated in Fig. 4.
These decreases in the 1 -day $\mathrm{T} / \mathrm{M}$ ratios cannot be explained on the basis of different choroid plexus weights. For example, the average weights of the choroid plexuses in group II and group III (the two groups with the greatest weight difference) are 6.54士 $0.21 \mathrm{mg}$ (SEM; $n=14$ ) and $7.82 \pm 0.61 \mathrm{mg}$ (SEM; $n=16$ ), respectively, with $P>0.05$ (Student's $t$ test).

The percent of penicillin unbound in the CSF of rabbits inoculated intracisternally the previous day with $5 \times 10^{7} \mathrm{H}$. $f u$. was $76 \pm 6 \%$ (SEM). This percentage is the average of 11 microbiological and 4 radiochemical determinations in the 3 sets of 11 rabbits total. These findings differ from those of Ruedy (19), who suggested that $90 \%$ of penicillin was unbound in meningitic CSF. However, Ruedy did not directly measure this percentage.

Clearance studies. The results of the clearance 
studies are shown in Table III. With the ventriculocisternal perfusion technique, no significant difference was observed in the clearance of penicillin from the CSF compartment or in the rate of CSF production. However, in unanesthetized rabbits, there was a significant difference $(P<0.05)$ in the removal of penicillin compared to inulin in control versus $H$. flu.-inoculated rabbits. When $\left[{ }^{14} \mathrm{C}\right]$ penicillin and $\left[{ }^{3} \mathrm{H}\right]$ inulin were injected simultaneously into the ventricles and the rabbit was allowed to awake before removal of CSF from the cisterna magna, the ratio of penicillin to inulin in CSF divided by the same ratio in the injectate and multiplied by 100 was $8.9 \pm 2.1 \%(\mathrm{SEM} ; n=5)$ in $H$. $f$. -inoculated rabbits versus $4.1 \pm 0.2 \%$ (SEM; $n=$ 5) in controls.

\section{DISCUSSION}

The model of resolving meningeal inflammation (induced by CSF inoculation of $H$. $f(u$.) used in our study would not be a satisfactory experimental model of $H$. flu. meningitis since the adult rabbits' nautral immunity prevented invasion and multiplication of the bacteria (2, 10). However, although this model is pathogenetically unrelated to nautrally acquired meningitis, penicillin dis-

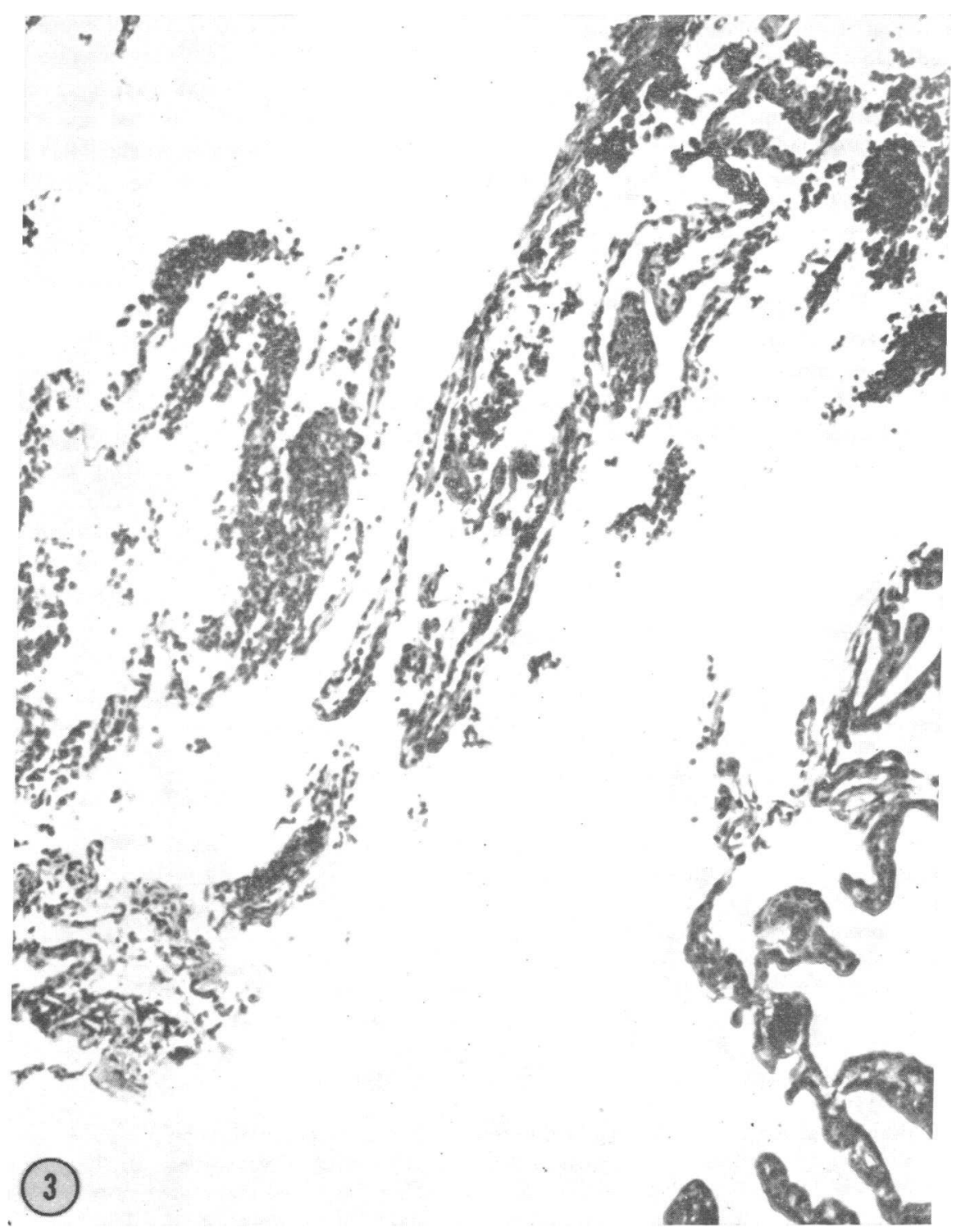

FIGURE 3 The fourth ventricular choroid plexus was obtained $9 \mathrm{~h}$ after the intracisternal inoculation of approximately $10^{8} \mathrm{~S}$. aureus. The choroid plexus was significantly infiltrated with inflammatory cells. Hematoxylin and eosin stain. $\times 230$. 
TABLE I

Penetration of Mannitol into CSF and Choroid Plexus of Unanesthetized Rabbits*

\begin{tabular}{cccc}
\hline$n$ & $\begin{array}{c}\text { Status of } \\
\text { rabbits }\end{array}$ & CSF $\ddagger$ & Choroid plexus $\ddagger$ \\
\hline 6 & Control & $0.17 \pm 0.01 \S$ & $0.57 \pm 0.05$ \\
5 & H.fu. & $0.48 \pm 0.07$ & $0.45 \pm 0.06$ \\
\hline
\end{tabular}

* Rabbits, inoculated intracisternally with either $H$. $f u$. or PBS (control) the previous day, were infused with $30 \mu \mathrm{Ci}$ $\left[{ }^{3} \mathrm{H}\right]$ mannitol i.v. over $3 \mathrm{~h}$. At that time, samples of plasma and choroid plexus were obtained and analyzed for $\left[{ }^{3} \mathrm{H}\right]$ mannitol.

¥ Values given are ratios of tissue to plasma mannitol at $3 \mathrm{~h}$. $\S$ Values are means $\pm \mathrm{SEM} ; n=$ number of animals.

$\|$ Value designated by $\|$ is statistically different from controls with $P<0.01$ (Student's $t$ test as modified by Chochran for comparisons of means with unequal variances (30).

T Plasma mannitol levels were $1.02 \pm 0.21 \times 10^{5} \mathrm{dpm} / \mathrm{ml}$ $(\mathrm{SEM} ; n=5)$ and $1.09 \pm 0.09 \times 10^{5} \mathrm{dpm} / \mathrm{ml}(\mathrm{SEM} ; n=6)$ in the $H$. $f u$. and control rabbits, respectively.

tributions comparable to those seen in naturally acquired human meningitis were observed. Thus, this model was useful for the purpose intended (Table II) $(20,21)$. Similar results have been noted by others in other models of experimental meningitis $(2-4,19,22)$. Moreover, the increased penetration of mannitol into the CSF compartment in this model (Table I) was similar to that seen in experimental pneumococcal meningitis in dogs (1). This altered distribution is consistent with inflammatory changes in the membranes that comprise the blood-CSF barrier $(1,2)$ as illustrated in Figs. 1 and 2 .

However, penicillin, unlike mannitol, is actively transported from the CSF compartment (7-9). The ratio of the efflux and influx of penicillin from and into the $\mathrm{CSF}$ compartment determines $\mathrm{CSF} /$ plasma penicillin ratios $(6,9,18)$. It should be noted that the efflux of penicillin is more than 10 times the influx (9). In vivo in normal rabbits and dogs, it is possible to block this efflux mechanism with probenecid $(7,9)$. The locus of the efflux process resides, in part, in the choroid plexus $(9,23)$. In this study, we have shown that the inflamed choroid plexuses in both the rapidly fatal ( $S$. aureus) and resolving $(H . f u$.) model were unable to concentrate penicillin as effectively as choroid plexuses from controls. This depression was observed even though the choroid plexuses were placed in $3 \mathrm{ml}$ of incubation medium that could have washed out or diluted possible toxic factors. However, the choroid plexuses isolated from rabbits 5 days after intracisternal inoculation of $H$. $f l u$. showed total recovery of ability to concentrate penicillin. Moreover, even in the moribund, bacteremic rabbits with $S$. aureus meningitis, the choroid plexuses still retained a significant ability to take up penicillin and presumably still actively transport penicillin from CSF to blood. Evidence that active transport of penicillin still exists in vivo in rabbits with $S$. aureus meningitis can be inferred from data by Lithander and Lithander, and Ruedy $(11,19)$.

In the clearance studies employing ventriculocisternal perfusions, no significant difference was found in the clearance of penicillin from the perfused CSF compart-

TABLE II

Penetration of Penicillin into CSF of H. flu.-Inoculated Rabbits with and without Probenecid Pretreatment*

\begin{tabular}{lllccccc}
\hline Group & $n$ & Status of rabbits & $\begin{array}{c}\text { Unbound } \\
\text { plasma } \\
\text { penicillin }\end{array}$ & $\begin{array}{c}\text { Total plasma } \\
\text { penicillin }\end{array}$ & $\begin{array}{c}\text { Unbound } \\
\text { plasma } \\
\text { penicillin }\end{array}$ & $\begin{array}{c}\text { Total CSF } \\
\text { penicillin }\end{array}$ & $\begin{array}{c}\text { Total CSF penicillin } \\
\text { Unbound plasma penicillin }\end{array} \times 100$ \\
\hline & & & $\mu g / m l$ & $\mu g / m l$ & $\%$ & $\mu g / m l$ & $\%$ \\
I & 5 & Control & $8.0 \pm 1.1 \S$ & - & - & $0.19 \pm 0.04$ & $2.6 \pm 0.7$ \\
II & 4 & H. $f$ lu. & $5.6 \pm 1.2$ & $15.1 \pm 2.8$ & $38.0 \pm 6.0$ & $0.58 \pm 0.12 \|$ & $11.0 \pm 2.5 \|$ \\
III & 4 & H. $f l u$. probenecid $\ddagger$ & $6.6 \pm 1.4$ & $14.9 \pm 2.8$ & $45.8 \pm 5.1$ & $2.21 \pm 0.27$ & $35.4 \pm 4.6$ \\
\hline
\end{tabular}

* On the day before the experiment, rabbits were inoculated intracisternally with $H$. $f u$. or PBS (controls). After saline pre treatment in group I and II or probenecid pretreatment $(200 \mathrm{mg} / \mathrm{kg}$ i.p.) in group III, rabbits were infused with $100 \mathrm{mg} / \mathrm{kg}$ penicillin i.v. over $3 \mathrm{~h}$. At that time, samples of CSF, plasma, and ultrafiltrate of plasma were obtained and analyzed for penicillin. $\ddagger$ Probenecid pretreated rabbits were infused with $33 \mathrm{mg} / \mathrm{kg}$ penicillin over $3 \mathrm{~h}$.

$\$$ Values are means \pm SEM. $n=$ number of animals.

|| Values in group II designated by $\|$ are statistically different from comparable values in groups I and III with $P<0.05$ (Student's $t$ Test as modified by Cochran for comparison of means with unequal variances (30). If a log-normal distribution for CSF penicillin as suggested by Lithander and Lithander (11) rather than a normal distribution is assumed, the variances for the logarithms of the CSF penicillin become approximately equal. This assumption of a log-normal distribution allows Scheffe's method for multiple comparisons in the Gaussian analysis of variance to be applied to the logarithms of the CSF penicillin values, since the logarithms of the observations would be normally distributed (31). By Scheffe's analysis, the CSF penicillin values in any group differ from those in any other group with $P<0.05$. 


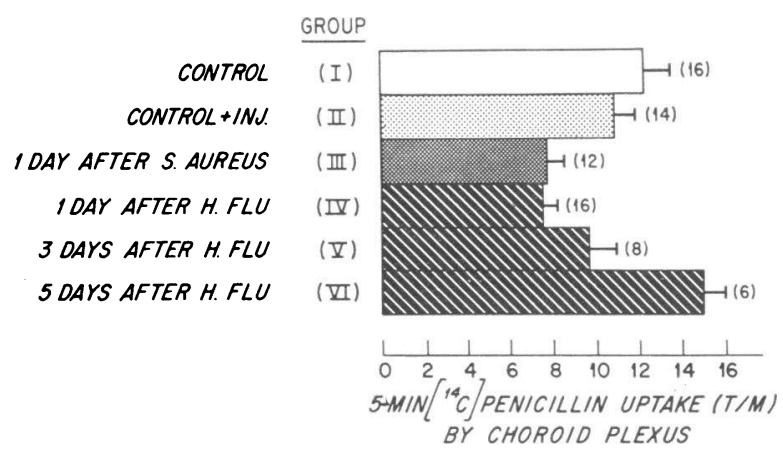

FIGURE 4 5-min in vitro uptakes of $\left[{ }^{14} \mathrm{C}\right]$ penicillin, expressed as a $\mathrm{T} / \mathrm{M}$ ratio, by the choroid plexuses of six groups of rabbits. Group I rabbits were uninjected controls. Group II rabbits were injected intracisternally with PBS; group III rabbits were inoculated intracisternally with approximately $10^{8} S$. aureus; group IV, V, and VI rabbits were injected intracisternally with $5 \times 10^{7} H$. flu. On the day after CSF withdrawal and/or intracisternal inoculation, the 5 -min in vitro $\mathrm{T} / \mathrm{M}$ ratio for $\left[{ }^{14} \mathrm{C}\right]$ penicillin was obtained for the choroid plexuses of rabbits in groups I, II, and IV. Group III rabbits were sacrificed $12 \mathrm{~h}$ after inoculation. The 5-min $\mathrm{T} / \mathrm{M}$ ratios for the choroid plexuses of rabbits in groups $\mathrm{V}$ and VI were obtained 3 and 5 days after the intracisternal inoculation, respectively. The concentration of penicillin in the medium was $0.001 \mathrm{mM}$. Values are means \pm SEM with the number of choroid plexuses in parentheses. The means obtained for the rabbits in groups III and IV are statistically different from the means in groups I and VI with $P<0.05$ by Scheffe's method for multiple comparisons in the Gaussian analysis of variance (29). Without the assumption of a normal distribution, the medians of the six groups differ significantly by the nonparametric Kruskel-Wallis one-way analysis of variance with $H=25.8$ and $P<0.005$ (29).

ment or in the rate of CSF formation. These findings could be accounted for if there were indeed no differences, or, if the differences were reversed or obscured during the perfusion before the steady state clearances and CSF formation rates were obtained. Anesthesia is known to depress clearance from the $\operatorname{CSF}(24,25)$ and could have obscured a significant difference. Secondly, it is possible that the washout and dilution of the purulent CSF by the perfusate from the CSF compartment may have partially reversed the depression of the choroid plexus transport system. We believe that either anesthesia or washout, or both, could explain the results of the ventriculocisternal perfusions. This hypothesis was supported by the finding that when the disappearance of penicillin was measured by perturbing the CSF compartment less (viz., by injecting the $\left[{ }^{14} \mathrm{C}\right]$ penicillin into the left lateral ventricle and then allowing the animal to become conscious before a cisternal sample was obtained), the disappearance of penicillin relative to inulin in the $H$. $f u$.-inoculated animals was significantly less than in controls (Table III). This difference could not he explained by penicillin binding in the CSF alone. Moreover, since penicillin is a considerably smaller molecule than inulin ( $\mathrm{mol} \mathrm{wt}=374$ versus mol wt $=5,000$ ), it is probable, on the basis of size alone, that penicillin would diffuse more rapidly than inulin through inflamed meninges (26). Prockop and Fishman (1) showed that inulin, after cisternal injection of pneumococci, left the CSF $40 \%$ faster $(P<0.05)$ in meningitic as compared to control dogs (data combined from Tables III and VII, in ref. 1). Mannitol was cleared at a $90 \%$ faster rate $(P<0.05)$ in meningitic dogs (1). Quantitative conclusions could not be drawn because CSF formation and bulk flow were not measured. However, it was possible to conclude that the disappearance of mannitol, which normally leaves the CSF compartment faster than inulin, was disproportionately increased in meningitic dogs (1).

Cooper, Beaty, Oppenheimer, Goodner, and Petersdorf (27) attempted to measure CSF formation in dogs with experimental pneumococcal meningitis. They concluded, with reservations, that $\mathrm{CSF}$ formation was not

TABLE III

Clearance of Penicillin from the CSF in Control and II. Flu. Inoculated Rabbits*

\begin{tabular}{|c|c|c|c|}
\hline$x$ & $\begin{array}{l}\text { Status of } \\
\text { rabbits }\end{array}$ & $\begin{array}{c}\text { CSF } \\
\text { formation }\end{array}$ & $\begin{array}{l}\text { Penicillin } \\
\text { clearance }\end{array}$ \\
\hline & & $m l m i n$ & $m l m i n$ \\
\hline \multicolumn{4}{|c|}{ Series one. Ventriculocisternal perfusions } \\
\hline 4 & Control & $0.007 \pm 0.001_{t}^{+}$ & $0.014 \pm 0.002$ \\
\hline 6 & $H . f l u$. & $0.006 \pm 0.002$ & $0.011 \pm 0.003$ \\
\hline$x$ & $\begin{array}{l}\text { Status of } \\
\text { rabbit: }\end{array}$ & $\begin{array}{l}\text { Residual } \\
\text { cisternal } \\
\text { penicillin! }\end{array}$ & $\begin{array}{c}\text { Inulin in } \\
\text { cisterna magna }\end{array}$ \\
\hline & & $\%$ & $\mathrm{dpm} / \mathrm{ml} \times 10^{-6}$ \\
\hline
\end{tabular}

Series two. Cisternal sampling after ventricular injection
Control
$4.1 \pm 0.2$
$0.86 \pm 0.19$
$5 \quad H . f u$.
$8.9 \pm 2.1 \S$
$0.81 \pm 0.14$

* The clearance of $\left[{ }^{14} \mathrm{C}\right]$ penicillin from the CSF was measured by two techniques in rabbits inoculated intracisternally with $H$. $f l u$. or PBS (controls) the previous day. In series one, the rabbits were subjected to ventriculocisternal perfusions to obtain the CSF formation rate as well as the penicillin clearance from the CSF. In series two, $\left[{ }^{14} \mathrm{C}\right]$ penicillin and $\left[{ }^{3} \mathrm{H}\right]$ inulin were injected into the lateral ventricle. After $2 \mathrm{~h}$, the rabbits were reanesthetized with thiopental and CSF was removed from the cisterna magna. The penicillin and inulin concentrations in the CSF were determined.

$\ddagger$ Values are means \pm SEM. $n=$ number of animals.

$\S$ Value designated by $\S$ is statistically different from controls with $P<0.05$ by Student's $t$ test as modified by Cochran for comparison of means with unequal variances (30).

II Residual cisternal penicillin was defined as the ratio of ${ }^{14} \mathrm{C} /{ }^{3} \mathrm{H}$ in the cisternal $\mathrm{CSF}$ sample divided by the ratio ${ }^{14} \mathrm{C} /{ }^{3} \mathrm{H}$ in the solution injected into the ventricle times 100. 
depressed in their model of meningitis. In this study, we also found no significant irreversible change in CSF formation rates in $H$. fur.-inoculated versus control rabbits using the standard ventriculocisternal perfusion technique.

The depression of carrier-mediated penicillin transport reported in this study is similar to the depression of carrier-mediated glucose transport previously observed in experimental meningitis $(1,25)$. Our results differ in that glucose is transported by carrier-mediated facilitated diffusion $(1,27)$, whereas penicillin is actively transported against a gradient (7-9). Moreover, this depression of carrier-mediated glucose transport into CSF was exceeded by an increase in nonspecific permeability to glucose (1). In the case of penicillin, the effects of a presumed increase in nonspecific permeability of the blood-CSF barrier to penicillin, combined with the depression of active transport out of CSF, jointly increase CSF penicillin levels. With resolution of the meningitis, presumably the depression of the choroid plexus efflux transport system decreases (Fig. 4), and a decrease in the permeability of the blood-CSF membranes also occurs with a resultant decrease in CSF penicillin levels toward those seen in normal animals.

The exact factor ( $s$ ) that depress the carrier-mediated transport systems in the choroid plexus are not known. Possibilities include CSF acidosis, bacterial toxins, inflammatory cell products, and increased CSF lactate. It is worth noting that the changes in the $H$. $f u$. model employed in this study, as well as in human meningitis, are not irreversible (20). Moreover, in this model of meningeal inflammation, the penicillin transport system from CSF to blood was only partially depressed at $18 \mathrm{~h}$ after inoculation and could be further depressed with probenecid (Table II).

The implications for human disease of this study are several. Implicit in these speculations are the assumptions that penicillin distributions in naturally acquired human meningitis are similar to those observed in the models presented in this study $(20,21)$ and, secondly, that the pathophysiologic mechanisms are comparable. If these assumptions are correct, then:

(a) In human meningitis with a large amount of protein exudation, significant amounts of penicillin binding by the CSF may occur. Presumably, bound penicillin is not active (28), and CSF penicillin levels might therefore be misleading. It has been suggested that certain penicillins, like ampicillin, which tend to be less bound $(11,31)$, might therefore have significant advantages in human meningitis. This would be true only if the less bound penicillins were as potent as penicillin $\mathrm{G}$, and achieved the same concentrations in the vicinity of the organisms. (b) The depression in the choroid plexus transport systems may not always be related to the severity of the clinical state, as shown in our $S$. aureus experimental model. Other factors (as bacteremia) may contribute to the clinical state and ultimate outcome. Moreover, in humans, fatal meningitis may not necessarily be associated with high CSF levels of penicillins (20).

(c) Finally, it may be possible, in human meningitis, to depress further the choroid plexus' penicillin transport system with probenecid and thereby increase significantly CSF penicillin levels during the acute as well as the resolving phase of meningitis (9). Several reservations to this mode of therapy have been previously discussed (9).

One further reservation would be the effects of probenecid on the concentration of penicillin in brain and choroid plexus. These concentrations were not measured in this study. In another study, Fishman (7) showed that probenecid increased $\left[{ }^{14} \mathrm{C}\right]$ penicillin levels in rat brain in both low and high dose penicillin experiments. However, the increase in penicillin levels in brain was not proportional to the increase in ${ }^{14} \mathrm{C}$ levels in plasma. Fishman postulated that high dose $(250 \mathrm{mg} / \mathrm{kg})$ probenecid might depress penicillin entry into brain. However, Lithander and Lithander (11) showed that increasing the plasma levels of penicillin alone depressed the ratio of brain to plasma penicillin. This was also suggested by Fishman (Table VII, ref. 7). Whether these possible effects on brain penicillin levels might militate against increasing CSF levels of penicillin in meningitis with probenecid remains to be established.

\section{ACKNOWLEDGMENTS}

The authors wish to thank Dr. Antone Medeiros for his gift of the Sarcina lutea organisms, Dr. Arnold Smith for his gift of the Hemophilus influenzae organisms, and Drs. Robert Snodgrass and Norman Uretsky for their helpful suggestions.

This study was supported in part by National Institutes of Health Grants NS 05172; NS-HD 09704 and The Children's Hospital Medical Center Mental Retardation and Human Developmental Research Program Grant HD 03773.

\section{REFERENCES}

1. Prockop, L. D., and R. A. Fishman. 1968. Experimental pneumococcal meningitis. Arch. Neurol. 19: 449-463.

2. Harter, D. H., and R. G. Petersdorf. 1960. A consideration of the pathogenesis of bacterial meningitis: review of experimental and clinical studies. Yale J. Biol. Med. 32: $280-309$.

3. Lithander, A. 1965. The passage of parenteral ampicillin into the cerebrospinal fluid in Haemophilus influenzae meningitis. Acta Pathol. Microbiol. Scand. 64: 320-334.

4. Lithander, A., and B. Lithander. 1966. The passage of benzyl penicillin into the cerebrospinal fluid after administration in Haemophilus influenzae meningitis. Acta Pathol. Microbiol. Scand. 67: 569-572. 
5. Mehrtens, H. G., and H. F. West. 1917. The absorption of phenolsulphonphthalein from the subarachnoid space in diseases of the central nervous system. Arch. Intern. Med. 20: 575-585.

6. Pappenheimer, J. R., S. R. Heisey, and E. F. Jordan. 1961. Active transport of Diodrast and phenolsulfophthalein from cerebrospinal fluid to blood. Am. J. Physiol. 200: 1-10.

7. Fishman R. A. 1966. Blood-brain and CSF barriers to penicillin and related organic acids. Arch. Neurol. 15: 113-124.

8. Dixon, R. L., E. S. Owens, and D. P. Rall. 1969. Evidence of active transport of benzyl- ${ }^{14} \mathrm{C}$-penicillin from cerebrospinal fluid to blood. J. Pharm. Sci. 58: 1106-1109.

9. Spector, R., and A. V. Lorenzo. 1974. The effects of salicylate and probenecid on the cerebrospinal fluid transport of penicillin, aminosalicylic acid and iodide. $J$. Pharmacol. Exp. Ther. 188: 55-65.

10. Smith, A. L., D. H. Smith, D. R. Averill, Jr., J. Marino, and R. Moxon. 1973. Production of Hemophilus influenzae b. meningitis in infant rats by intraperitoneal inoculation. Infec. Immun. 8: 278-290.

11. Lithander, A., and B. Lithander. 1962. The passage of penicillin into the cerebrospinal fluid after parenteral administration in staphylococci meningitis. An experimental investigation on rabbits. Acta Pathol. Microbiol. Scand. 56: 435-450.

12. Davidsohn, I., and B. Henry. 1969. Clinical Diagnosis by Laboratory Methods. W. B. Saunders Company, Philadelphia. 14th edition. 48.

13. Grove, D. C., and W. A. Randall. 1955. Assay Methods for Antibiotics. Medical Examination Publishing Co., Inc., Flushing, N. Y.

14. Lorenzo, A. V., and R. Spector. 1973. Transport of salicylic acid by the choroid plexus in vitro. J. Pharmacol. Exp. Ther. 184 : 465-471.

15. Bollinger, H. R., M. Brenner, H. G. Gänshirt, H. K. Mangold, H. Seiler, E. Stahl, and D. Waldi. 1965. Thin-Layer Chromatography. Academic Press, Inc., New York. 315.

16. McGilvray, I. J., and R. D. Strickland. 1967. Detection and separation of penicillins by thin-layer chromatography. J. Pharm. Sci. 56: 77-79.

17. Spector, R., D. T. Korkin, and A. V. Lorenzo. 1972. A rapid method for the determination of salicylate binding using ultrafilters. J. Pharm. Pharmacol. 24: 786-789.
18. Spector, R., and A. V. Lorenzo. 1973. The transport and metabolism of salicylate in the central nervous system. In vivo studies. J. Pharmacol. Exp. Ther. 185: 276-286.

19. Ruedy, J. 1965. The concentrations of penicillins in the cerebrospinal fluid and brain of rabbits with experimental meningitis. Can. J. Physiol. Pharmacol. 43: 763: 772.

20. Thrupp, L. D., J. M. Leedom, D. Ivler, P. F. Wehrle, B. Portnoy, and A. W. Mathies. 1965. Ampicillin levels in the cerebrospinal fluid during treatment of bacterial meningitis. Antimicrob. Agents Chemother. 5: 206-213.

21. Mathies, A. W., Jr., J. M. Leedom, L. D. Thrupp, D. Ivler, B. Portnoy, and P. F. Wehrle. 1965. Experience with ampicillin in bacterial meningitis. Antimicrob. Agents Chemother. 5: 610-617.

22. Lithander, A. 1965. Benzyl penicillin and ampicillin in the cerebrospinal fluid during experimental Haemophilus influenzae meningitis. Antimicrob. Agents Chemother. 5: $435-438$

23. Cserr, H. F. 1971. Physiology of the choroid plexus. Physiol. Rev. 51: 273-311.

24. Aquilonius, S. M., and B. Winbladh. 1972. Cerebrospinal fluid clearance of choline and some other amines. Acta Physiol. Scand. 85 : 78-90.

25. Lorenzo, A. V., J. P. Hammerstad, and R. W. P. Cutler. 1968. The effect of anesthetic agents in the cerebrospinal fluid clearance of ${ }^{35} \mathrm{~S}$ sulphate and ${ }^{{ }^{22} \mathrm{I}}$ iodide. Biochem. Pharmacol. 17 : 1279-1283.

26. Welch, K., K. Sadler, and R. Hendee. 1970. Cooperative phenomena in the permeation of sugars through the lining epithelium of choroid plexus. Brain Res. 19: $465-482$.

27. Cooper, A. J., H. N. Beaty, S. P. Oppenheimer, C. J. Goodner, and R. G. Petersdorf. 1968. Studies on the pathogenesis of meningitis. VII. Glucose transport and spinal fluid production in experimental pneumococcal meningitis. J. Lab. Clin. Med. 71: 473-483.

28. Barlow, C. F. 1964. Clinical aspects of the blood-brain barrier. Annu. Rev. Med. 15 : 187-202.

29. Colquhoun, D. 1971. Lectures on Biostatistics. An introduction to statistics with applications in biology and medicine. Clarendon Press, Oxford. 210.

30. Snedecor, G. W., and W. G. Cochran. 1967. Statistical Methods. Iowa State University Press, Ames, Iowa. 6th edition. 114.

31. Kunin, C. M. 1965. Clinical pharmacology of the new penicillins. Clin. Pharmacol. Ther. 7: 180-188. 